\title{
Cobertura de las TIC en la educación básica rural y urbana en Colombia ${ }^{1}$ - 2018
}

\section{ICT coverage in rural and urban basic education in Colombia-2018}

\author{
Valeria Cruz - Carbonell ${ }^{1}$, Ángel Fabián Hernández - Arias², Adriana Carolina Silva - \\ Arias $^{3}$
}

\begin{abstract}
${ }^{1}$ Estudiante del Programa de Economía, Integrante del Semillero de Estudios Económicos, Universidad Militar Nueva Granada, Colombia, ORCID: https://orcid.org/0000-0002-7955-5257, E-mail: u2102074@unimilitar.edu.co

${ }^{2}$ Estudiante del Programa de Economía, Integrante del Semillero de Estudios Económicos, Universidad Militar Nueva Granada, Colombia, ORCID: https://orcid.org/0000-0002-6179-1112, E-mail: u2102114@unimilitar.edu.co

${ }^{3}$ Tutora del Proyecto de Iniciación Científica, Líder del Semillero de Estudios Económicos, Profesora del Programa de Economía, Universidad Militar Nueva Granada, Colombia, ORCID: https://orcid.org/0000-0003-1610-881X,

E-mail: adriana.silva@unimilitar.edu.co
\end{abstract}

Cómo citar: Cruz - Carbonell, V., Hernández - Arias, Ángel F., \& Silva - Arias, A. C. (2020). Cobertura de las TIC en la educación básica rural y urbana en Colombia. Revista Científica Profundidad Construyendo Futuro, 13(13), 3948. https://doi.org/10.22463/24221783.2578

Recibido: 25 de Marzo de 2019 / Aprobado: 18 de Mayo de 2019

\begin{abstract}
Resumen
Esta investigación describe la cobertura y otras características relacionadas con el acceso a los bienes y servicios propios de Tecnologías de la Información y la Comunicación (TIC) en las sedes educativas ubicadas en las zonas rurales y urbanas de Colombia. El universo de estudio se delimitó a centros educativos que permiten el acceso a los grados de primaria y secundaria. Los datos utilizados provienen del Censo de Educación Formal (EDUC) del Departamento Administrativo Nacional de Estadística (DANE). El principal objetivo es analizar las desigualdades correspondientes a la cobertura de estos bienes y servicios en dichas instituciones. Los principales hallazgos de la investigación demuestran las brechas significativas de su implementación en la educación, diferencias que deben ser contrarrestadas a partir de la formulación y ejecución de políticas educativas que favorezcan el acceso a estas herramientas, principalmente en el sector rural, con el propósito de contrarrestar las diferencias en términos de calidad y desempeño educativo en Colombia.
\end{abstract}

Palabra clave: Desigualdad de oportunidades, Educación, Tecnología, Segregación Espacial, Cobertura.

\begin{abstract}
This research describes coverage and other characteristics related to access to Information and Communication Technologies (ICT)'s goods and services in educational centers located in rural and urban areas of Colombia. The universe of study was delimited to educational centers that allow access to the primary and secondary level. The data used comes from the Formal Education Census (EDUC) of the National Administrative Department of Statistics (DANE). The main objective is to highlight the inequalities related to the coverage of these goods and services in

\footnotetext{
${ }^{1}$ Producto derivado del Proyecto de Iniciación Científica PIC-ECO-3310 financiado por la Vicerrectoría de Investigaciones de la Universidad Militar Nueva Granada - Vigencia 2020-2, titulado "Cobertura de las TIC en la educación básica rural y urbana en Colombia-2018", como parte de los productos asociados al proyecto IMP-ECO 3116 financiado por la Vicerrectoría de Investigaciones de la Universidad Militar Nueva Granada - Vigencia 2020/2022. Una versión preliminar de este documento fue presentada en el VI Congreso Internacional DOSSIER realizado el 10 y 11 de junio de 2020 por plataforma virtual.
} 
various institutions. The main findings of the research found the gaps of their implementation in education, these differences must be counteracted from the formulation and execution of educational policies that favor access to these tools, mainly in the rural sector, in order to counteract the differences in terms of quality and educational performance Colombia.

Keyword: Inequality of opportunity, Education, Technology, Rural Area, Spatial Segregation, Enrolment.

\section{Introducción}

La educación es un factor que contribuye a reducir las desigualdades sociales y económicas presentes en una sociedad (Fernández, 2014). Así mismo, es una herramienta que permite el desarrollo integral de los individuos y posibilita el ejercicio de otros derechos fundamentales (Roemer y Trannoy, 2016). En Colombia, el sistema educativo puede llegar a agudizar las desigualdades sociales debido a la ausencia de equidad en las condiciones de infraestructura y procesos de aprendizaje (Gento et al., 2018)

En la literatura se considera que el uso de nuevas tecnologías es un aspecto clave en los procesos de formación académica, dado que facilita el acceso al conocimiento e impacta positivamente en el sistema educativo (MinTIC, 2020). A su vez, los bienes y servicios TIC son herramientas que permiten aumentar la cantidad de población atendida, proporcionando una mejora continua en la cobertura educativa (Alegre, 2015).

Investigaciones previas han reconocido la importancia y el alcance de los bienes y servicios TIC para mejorar los procesos organizacionales y el desarrollo social. Por lo tanto, las organizaciones pertenecientes al sector educativo incorporaron el uso de estas herramientas con el propósito de implementar nuevas estrategias pedagógicas en la enseñanza tradicional, con el fin de propender por una mayor calidad y pertinencia en los programas educativos (Ruiz, 2017).
No obstante, el acceso a estos bienes y servicios se ha dado de manera desigual ocasionando un aumento en las brechas de desempeño y logro académico (Murphy \& Sunthonkanokpong, 2019). Adicionalmente, Espinosa et al. (2013) señalan que la ausencia de recursos pedagógicos es una de las principales barreras para lograr una adecuada movilidad social.

Para el caso de Colombia, las Tecnologías de la Información y la Comunicación (TIC), han sido un factor importante en la formulación de proyectos educativos para el cierre de brechas educacionales (MinTIC, 2020). Sin embargo, existe un número significativo de instituciones educativas que no acceden a recursos tecnológicos para implementar nuevas metodologías de aprendizaje, principalmente en las zonas rurales del país.

En el boletín técnico de educación formal realizado por el DANE (2019), se evidenciaron las diferencias en el acceso a servicios, como el internet. Para el 2018, el $89.2 \%$ de las sedes educativas ubicadas en las zonas urbanas tuvieron acceso a este servicio, mientras que en el sector rural tan solo el $26.9 \%$ de las sedes educativas contaron con él.

En relación con lo anterior, es importante reconocer la necesidad de incorporar los bienes y servicios TIC en la pedagogía educativa, debido a los múltiples impactos positivos que generan en los indicadores 
educativos y en el desempeño de los estudiantes.

Respecto a los indicadores educativos, el Consejo privado de competitividad (2019) reveló que para el 2018 la cobertura neta para el nivel de primaria y secundaria fue de $82 \%$ y $72 \%$, respectivamente. Dicho indicador podría mejorarse con un acceso incluyente y equitativo a los bienes y servicios TIC en las sedes educativas y en los hogares de los colombianos.

Según UNESCO (2008), el uso adecuado de las TIC puede contribuir no solo a mejorar los indicadores de acceso y calidad educativa, sino también a fomentar el desarrollo profesional de los docentes y demás actores del sistema educativo de manera eficiente (Flórez, 2016).

A pesar de la importancia que tiene el acceso a bienes y servicios TIC en el sector educativo, es escasa la literatura enfocada en analizar este tema y comprender las disparidades que se presentan entre zonas y niveles educativos. Por lo tanto, se considera relevante analizar el acceso y la cobertura principalmente en las sedes educativas, las cuales son actores fundamentales en la formación de capital humano.

Debido a lo anterior, esta investigación tiene por objetivo analizar los niveles de cobertura y uso de las TIC en las instituciones de educación básica rurales y urbanas en Colombia para el 2018. A partir de los hallazgos se proponen recomendaciones que permitan disminuir las desigualdades en términos educativos.

Esta investigación está organizada de la siguiente manera. Primero se presenta una revisión de literatura, donde se contextualizan algunos conceptos relacionados con el tema y aportes de otras investigaciones acerca de las condiciones de acceso y cobertura de bienes TIC en Colombia.

Seguido de esto se presenta la metodología utilizada para la investigación, la cual tendrá un enfoque cuantitativo a partir de un análisis descriptivo de los datos disponibles para el desarrollo de la investigación. Por último, se presentan los resultados, discusión y conclusiones a partir de los hallazgos.

\section{Marco teórico}

La incidencia de educación en la sociedad puede analizarse bajo dos enfoques. El primero, denominado "visión de corte liberal", indica que la educación favorece de forma equitativa a todos aquellos que participen de ella, de modo que los individuos adquieren la facultad de ser autónomos y activos en la sociedad. El segundo enfoque sostiene que la educación reproduce las desigualdades sociales, ya que el sistema educativo suele favorecer a aquellos estudiantes con mejores condiciones de vida, marginando a estudiantes de clases desfavorecidas a una pedagogía mediocre y carente de visión (Espinosa et al., 2015).

En relación con la segunda visión expuesta, Otero-Bahamon (2019) encuentra que por lo general los estudiantes en condiciones económicas desfavorables asisten a sedes educativas que carecen de recursos pedagógicos y herramientas tecnológicas, lo que agudiza las desigualdades educativas.

Respecto a las Tecnologías de la Información y Comunicación (TIC), Graells (2000) aclara que no solamente se refieren a aquellas asociadas a la multimedia y telemática, sino también a los medios de comunicación social o "mass media" y los 
medios de comunicación con soporte tecnológico tradicional como el fax o el teléfono. Las herramientas TIC están conformadas por computadores, celulares y tabletas. Del mismo modo, los servicios TIC están conformados por electricidad, radio, línea telefónica, televisión e internet (DANE, 2019b).

Las tecnologías de la información y la comunicación han transformado los procesos de transmisión de conocimiento y han generado cambios significativos en términos educativos (Cuenca, 2016). La implementación de las TIC en la educación supone no solo una herramienta gestora de evolución en la pedagogía, sino todo un reto tanto para quienes experimentan el cambio como para quienes lo ejercen dentro y fuera de las aulas (García, 2010).

Tal como ocurre en la mayoría de los países de América Latina, Colombia presenta inconvenientes en la incorporación de las tecnologías en los distintos contextos educativos. Uno de los inconvenientes se relaciona con el uso de computadores en áreas urbanas y rurales. Para el 2015, mientras que en las zonas urbanas el $69 \%$ de los jóvenes usaron computador, en las áreas rurales solo lo hizo el $36.7 \%$, evidenciando una brecha significativa entre ambas zonas (Alegre, 2015).

De acuerdo con Hernández (2017), el impacto de las TIC en la educación y la utilización de estas tecnologías va más allá de la simple implementación de novedosas herramientas, también es un replanteamiento en la consolidación del conocimiento.

MinTIC (2020) presentó una investigación realizada por el Centro Nacional de Consultoría en el 2015, la cual revisaba el comportamiento y avance que presentaron los estudiantes y las sedes educativas oficiales en Colombia, luego de implementarse la propuesta pedagógica de "Computadores para Educar". Este estudio mostró que transcurrido un año desde que se entregaran las herramientas tecnológicas en las sedes, los indicadores de desempeño y las pruebas estandarizadas presentaron una mejora.

El sector rural colombiano durante décadas ha experimentado mayores dificultades para consolidar un adecuado desarrollo en la calidad de vida de quienes habitan en estas zonas. Lo anterior producto del bajo nivel de escolaridad alcanzado por los pobladores de estas zonas, sumado al abandono estatal en muchas regiones y la violencia crónica. Para mitigar esta condición, se hace necesario considerar las TIC como un elemento más de clase, así como el lápiz y el papel (Duarte et al., 2017).

Respecto a los indicadores de logro académico, Martínez et al. (2016) evidenciaron que el sistema educativo colombiano presentó una tasa de permanencia en las zonas urbanas del $82 \%$ y en las rurales del $48 \%$ para el 2014. Del mismo modo, en este estudio se pudo evidenciar que los resultados de las pruebas estandarizadas Saber $5^{\circ}, 9^{\circ}$ y $11^{\circ}$ en las zonas rurales fueron inferiores a los resultados en las zonas urbanas.

La situación de las zonas rurales refleja las brechas educacionales presentes entre estas dos áreas, así como el reto que representa el cierre de estas desigualdades sociales. Es fundamental aumentar la calidad $y$ pertinencia educativa con el acceso a bienes tecnológicos.

Existen grandes desigualdades en la utilización de TIC en las sedes educativas de las zonas rurales y urbanas. En las zonas urbanas colombianas la utilización de internet 
para la educación alcanzó el 89.2\%, mientras que en el sector rural su implementación apenas alcanzó el 26.9\% (DANE, 2019a).

\section{Metodología}

La presente investigación pretende realizar un análisis cuantitativo de tipo descriptivo acerca de la cobertura y otras características relacionadas con el acceso a los bienes y servicios TIC en las instituciones académicas que imparten el nivel educativo básico en las zonas rurales y urbanas en Colombia. El universo de estudio de esta investigación se compone de 47,120 sedes educativas ubicadas en dichas zonas que reportaron información en el censo de Educación formal (EDUC) realizado por el Departamento Nacional de Estadística (DANE).

Del mismo modo, el año de estudio es el 2018, debido a que es el periodo más reciente disponible tanto en el censo EDUC como de otras fuentes de información cuantitativa, tales como los boletines técnicos proporcionados por el DANE en donde se exponen algunas cifras en relación con las características del sistema educativo y cobertura en bienes tecnológicos en dicho año, así como cifras proporcionadas por el Ministerio de Tecnologías de la Información y las Comunicaciones - MinTIC y el Ministerio de Educación - MinEduc, las cuales fueron insumo para el desarrollo de esta investigación.

Para el correspondiente análisis de la base de datos EDUC, se utilizó el paquete de software estadístico Stata 15.1, para delimitar el universo de estudio y producir los estadísticos descriptivos. En total se analizaron doce (12) de las doscientas trece (213) variables disponibles en el censo educativo.
Las variables analizadas corresponden al módulo de tenencia y uso de bienes y servicios TIC en las sedes educativas que ofrecieron el nivel académico básico, así como todas aquellas variables relacionadas con la frecuencia y distribución porcentual de estas características.

Dicha selección de datos se trabajó, en conjunto con el programa ArcMap 10.5, para la realización de una representación geográfica de la ausencia de conexión a internet en Colombia por departamento.

Sumado a esto, la información también fue organizada en dos tablas correspondientes al nivel educativo de básica primaria y secundaria, siendo el primer nivel conformado por los cursos $1^{\circ}, 2^{\circ}, 3^{\circ}, 4^{\circ}$ y $5^{\circ}$, y el segundo por los cursos $6^{\circ}, 7^{\circ}, 8^{\circ}$ y $9^{\circ}$. Lo anterior, para evidenciar diferencias en la cobertura de dichos bienes y servicios a través de la trayectoria educativa, que de acuerdo con la legislación colombiana constituye niveles en los que se debería tener cobertura universal.

Del mismo modo, se analizaron cifras proporcionadas por los boletines técnicos del DANE referentes a educación formal e indicadores básicos de tenencia y uso de Tecnologías de la Información y Comunicación - TIC en hogares y personas de 5 y más años.

\section{Resultados}

La figura 1 evidencia la distribución porcentual por departamento de sedes educativas que NO tuvieron acceso a internet durante el 2018.

Entre los departamentos con problemas para acceder al servicio de internet figuran 
Vichada, Vaupés, Chocó, Guaviare, Caquetá y Amazonas. Estas zonas geográficas han sufrido de mayores vulnerabilidades sociales, conflicto armado y menor urbanización (Centro Nacional de Memoria Histórica, 2018).

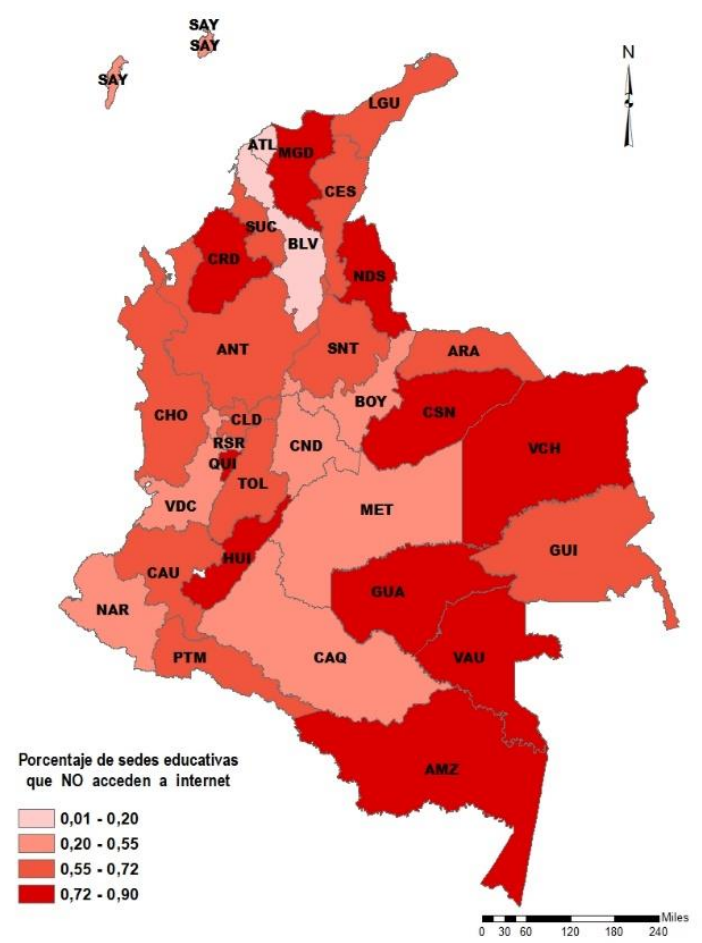

Figura 1. Porcentaje de sedes educativas por departamento que NO tuvieron conexión y acceso a internet. Fuente: EDUC 2018 -DANE. Elaboración propia.

Además de evidenciar las desigualdades territoriales, en general 7 de cada 10 sedes educativas de básica primaria se ubicaron en el sector rural. En el caso de las sedes educativas que imparten educación básica secundaria, 5 de cada 10 centros educativos estaban ubicados en las zonas urbanas, como se puede evidenciar en la figura 2.

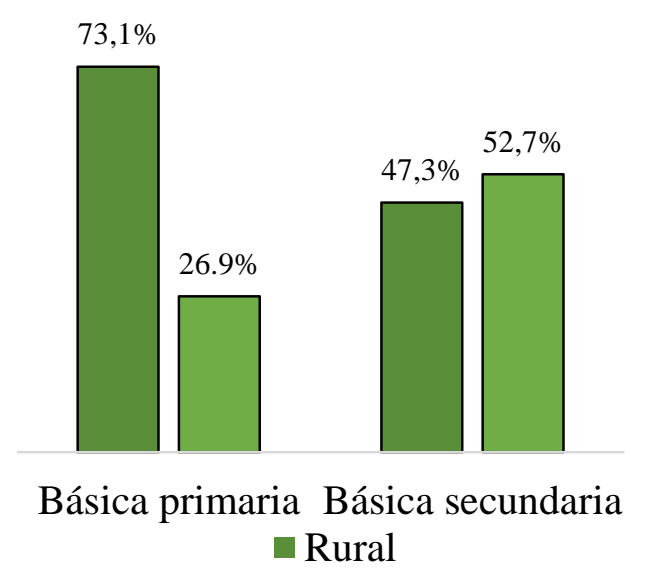

Figura 2. Distribución porcentual de sedes por nivel educativo básico y zona. Fuente: Basado en el Boletín Técnico EDUC 2018. Elaboración propia.

En la Tabla 1 se muestra el acceso a los servicios TIC en las sedes que ofrecen educación básica primaria. De esta forma, el 97.6\% de las sedes tuvieron servicio de electricidad en las zonas urbanas, este porcentaje de cobertura es mayor comparado con la proporción de las zonas rurales, las cuales contaban con un $83.5 \%$ de sedes con acceso a electricidad, constituyendo una diferencia de 14.1 puntos porcentuales.

Así mismo, el acceso al servicio de radio está notablemente rezagado tanto en las zonas urbanas como en las rurales, respectivamente con un $72.8 \%$ y un $89.4 \%$ de sedes que no tuvieron acceso a este servicio

Por otro lado, existen brechas en el acceso a servicio de internet, dado que en las zonas rurales solo el $23.3 \%$ de sedes contaban con acceso, mientras que en las zonas urbanas el $88.2 \%$ accedieron a internet; lo que se constituye en una profunda brecha en uso de tecnologías para el sistema educativo, ya que esta es una herramienta pedagógica necesaria, aún más en la actual crisis sanitaria. 
Tabla 1. Acceso a bienes y servicios en sedes educativas que dictan primaria

\begin{tabular}{cll}
\hline & Rural & Urbana \\
\hline $\begin{array}{c}\text { ¿Tiene electricidad? } \\
\text { No }\end{array}$ & 0.165 & 0.024 \\
Sí & 0.835 & 0.976 \\
¿Tiene radio? & & \\
No & 0.894 & 0.728 \\
Sí & 0.106 & 0.272 \\
¿Tiene internet? & & \\
No & 0.767 & 0.118 \\
Sí & 0.233 & 0.882 \\
¿Tiene equipo de & & \\
cómputo? & & \\
$\quad$ No & 0.208 & 0.045 \\
Sí & 0.792 & 0.955 \\
Número de alumnos & & \\
en la sede & & \\
$\quad$ & 34.238 & 232.375 \\
Número de docentes & $(56.777)$ & $(221.818)$ \\
en la sede & & \\
& 3.408 & 21.881 \\
& $(6.621)$ & $(22.420)$ \\
\hline Cantidad de sedes $N$ & 34337 & 12783 \\
\hline
\end{tabular}

Nota: Desviación estándar en paréntesis. Fuente: EDUC 2018 -DANE. Cálculos propios.

En la Tabla 2, se presenta el acceso a bienes y servicios TIC en las sedes que tienen cobertura para los grados de secundaria. Se puede evidenciar que existe una importante diferencia en lo que respecta a la cobertura de internet dependiendo de las zonas de análisis en las instituciones que ofrecen educación secundaria. Mientras que en el sector urbano el $95.1 \%$ de las sedes contaban con acceso a internet, en las zonas rurales esta cobertura apenas fue del $51.4 \%$.

En relación con el servicio de electricidad, el $11 \%$ de los planteles educativos ubicados en la zona rural no tenían acceso a electricidad y el $2.3 \%$ de las sedes en las zonas urbanas carecían del mismo. Este servicio es indispensable para el uso de bienes TIC y para el desarrollo de una educación de calidad.
Tabla 2. Acceso a bienes y servicios en sedes educativas que dictan secundaria

\begin{tabular}{lrr}
\hline & Rural & Urbana \\
\hline ¿Tiene electricidad? & & \\
No & 0.110 & 0.023 \\
Sí & 0.890 & 0.977 \\
¿Tiene radio? & & \\
No & 0.860 & 0.726 \\
Sí & 0.140 & 0.274 \\
¿Tiene internet? & & \\
$\quad$ No & 0.486 & 0.049 \\
$\quad$ Sí & 0.514 & 0.951 \\
¿Tiene equipo de & & \\
cómputo? & & \\
$\quad$ No & 0.078 & 0.020 \\
$\quad$ Sí & 0.922 & 0.980 \\
Número de alumnos en la & & \\
sede $\quad$ & & \\
$\quad$ & 99.047 & 334.358 \\
& $(110.101)$ & $(289.524)$
\end{tabular}

Número de docentes en la sede

\begin{tabular}{rrr} 
& 12.334 & 34.953 \\
& $(12.172)$ & $(25.263)$ \\
\hline Cantidad de sedes N & 6524 & 7217
\end{tabular}

Nota: Desviación estándar en paréntesis. Fuente: EDUC 2018 -DANE. Cálculos propios.

\section{Discusiones}

De acuerdo con la evidencia empírica expuesta anteriormente, el $73.1 \%$ de las sedes educativas de primaria se encontraban en la zona rural. De estas sedes, un $76.7 \%$ no contaban con servicio de internet, el $16.5 \%$ no tenían electricidad y un $89.4 \%$ no hicieron uso de la radio.

Por otro lado, el $26.9 \%$ de las sedes que dictaban primaria se encontraban en las zonas urbanas, de las cuales un $11.8 \%$ no accedieron a internet, un $2.4 \%$ no tenían electricidad y el $72.8 \%$ no hicieron uso de la radio. 
En consecuencia, existe una notoria segregación espacial en el acceso a bienes y servicios TIC en las sedes de zonas urbanas. La cobertura disminuye para la población rural a medida que transcurre la trayectoria educativa.

En relación con el nivel de secundaria, el $47.3 \%$ de las sedes educativas rurales tuvieron cobertura en este nivel. Así mismo, en cuanto al uso de los bienes y servicios TIC entre estas instituciones, el uso de internet correspondió al $51.4 \%$ de las sedes, el uso de electricidad al $89 \%$ y el uso de radio al $14 \%$.

Por otra parte, el $52.7 \%$ de las sedes que dictaban secundaria se ubicaron en zonas urbanas. La cobertura del servicio de internet en estas sedes fue de $95.1 \%$, la cobertura de electricidad fue del $97.7 \%$ y el uso de radio fue del $27.4 \%$.

Es preocupante observar que, desde un plano general, la relación existente entre la distribución de sedes por zona y la cobertura de bienes y servicios TIC en la educación reproduce las desigualdades en la cobertura educativa, las vulnerabilidades sociales, la segregación espacial y la baja calidad de vida en las zonas rurales.

\section{Conclusiones}

En la presente investigación se evidencia una brecha de cobertura de las TIC en las sedes educativas dependiente de la zona en la que se encuentren las instituciones académicas. De esta forma, aunque la ubicación geográfica de los alumnos no es más que una circunstancia, se constituye en una fuente relevante de desigualdad de oportunidades educativas.

Uno de los principales desafíos que se deben asumir para mejorar el desempeño y logro académico es reducir las desigualdades en términos de acceso a bienes y servicios TIC a lo largo del territorio, ya que han profundizado las brechas sociales y las oportunidades de movilidad social, especialmente en las zonas rurales.

Asimismo, se evidencia una diferencia de cobertura en los niveles de educación básica primaria y secundaria, la cual por derechos humanos debería ser universal. El rezago es más profundo en primaria y en las áreas rurales. Es recomendable consolidar acción para reducir las desigualdades y garantizar la plena cobertura, así como el acceso a los bienes y servicios TIC para aumentar el capital humano de los jóvenes colombianos a fin de mitigar los círculos viciosos de pobreza y desigualdad.

Por tanto, se considera relevante implementar proyectos educativos que permitan cerrar las brechas y los rezagos en el desempeño académico que se presentan entre las zonas urbanas y rurales, así como a través del ciclo educativo de los niveles de primaria a secundaria.

Adicionalmente, las estrategias educativas que se implementen deben tener un factor transversal relacionado con el uso de los bienes y servicios TIC en los procesos de aprendizaje con miras a mejorar la calidad y cobertura educativa en las zonas rurales del país.

Por tanto, se considera relevante la implementación de políticas educativas dirigidas a la cobertura e implementación de TIC para la educación en las zonas rurales. En la actualidad existen rezagos en dichas áreas que se relacionan con las brechas en el desempeño académico. Así, la implementación y el buen uso de las tecnologías de la información contribuiría a 
mitigar las diferencias de oportunidades educativas para los niños y jóvenes colombianos.

\section{Agradecimientos}

Al Semillero de Estudios Económicos de la Universidad Militar Nueva Granada, por apoyar la formación en investigación. En especial, agradecemos los comentarios y contribuciones realizadas por Deisy Galvis, asistente graduada del Grupo de Estudios Macroeconómicos.

\section{Referencias}

Alegre, S. (2015). Las nuevas generaciones de mujeres rurales como promotoras del cambio. IIPE-UNESCO. Recuperado de http://www.argentina.gob.ar/sites/defau 1t/files/mujeres_rurales_como_promoto ras_del_cambio.pdf

Centro Nacional de Memoria Histórica. (2018). Regiones y conflicto armado. Balance de la contribución del CNMH al esclarecimiento histórico (1st ed.).

Consejo privado de competitividad. (2019). Informe Nacional de Competitividad, 1325. Recuperado de www.puntoaparte.com.co

Cuenca, A. (2016). Desigualdad de oportunidades en Colombia: Impacto del origen social sobre el desempeño académico y los ingresos de graduados universitarios. Estudios Pedagógicos, 42(2), 69-93. https://doi.org/10.4067/S071807052016000200005

DANE. (2019a). Educación Formal (EDUC)2018. Departamento Administrativo Nacional de Estadística, 1-42.
Recuperado de http://www.dane.gov.co/files/investigac iones/boletines/educacion/bol_EDUC_1 8.pdf

DANE. (2019b). Indicadores básicos de tenencia y uso de Tecnologías-2018. Recuperado de http://www.dane.gov.co/files/investigac iones/boletines/tic/bol_tic_hogares_dep artamental_2018.pdf

Duarte, J. E., Fernández, F. H., \& García, R. A. (2017). Modelo de integración de las TIC en instituciones educativas con características rurales, Realidades, 24(2) $1-26$.

Espinosa, J., García, M., Jiménez, F., \& Parra, J. (2013). Separados y desiguales. Educación y clases sociales en Colombia. In Reino de los países Bajos. Recuperado de http://www.dejusticia.org/wpcontent/uploads/2017/04/fi_name_recur so_591.pdf

Espinosa, J., García, M., Jiménez, F., \& Parra, J. (2015). El papel social de la Educación. Recuperado de http://morferan600.blogspot.com/2015/ 09/foto-ricardo-pardo-el-papel-socialde.html

Fernández, A. (2014). Inclusión y equidad. Una educación que multiplica oportunidades. Entreculturas. http://www.entreculturas.org/sites/defau lt/files/inclusion_y_equidad_0.pdf

Flórez, J. (2016). La política pública de educación en Colombia: gestión del personal docente y reformas educativas globales en el caso colombiano. Academia \& Derecho, 13, 309. 
https://doi.org/10.18041/2215-

8944/academia.13.313

García, B. (2010). Modelos teóricos e indicadores de evaluación educativa. Revista Electrónica Sinéctica, 35, 1-21.

Gento, S., Gonzáles, R., Orden, V., \& Palomares, A. (2018). Integración de perspectivas sobre el perfil metodológico de una educación de calidad. Bordon, Revista de Pedagogía, $70(1)$, 25-42. https://doi.org/10.13042/Bordon.2017.5 0833

Graells, P. (2000). Las TIC y sus aportaciones a la sociedad. 7. Recuperado de http://www.sld.cu/galerias/pdf/sitios/sa ntiagodecuba/las_tic_y_sus_aportacion es_a_la_sociedad.pdf

Hernández, R. (2017). Impacto de las TIC en la educación: Retos y Perspectivas. Propósitos y Representaciones, 5(1), 325-347.

Martínez, S., Pertuz, M., \& Ramírez, J. (2016). La situación de la educación rural en Colombia, los desafíos del posconflicto y la transformación del campo. $1-14$. http://www.compartirpalabramaestra.or g/documentos/fedesarrollo_compartir/la -situacion-de-la-educacion-rural-encolombia-los-desafios-del-posconflictoy-la-trasformacion-del-campo.pdf

MinTIC. (2020). Las TIC siguen impactando positivamente la educación colombiana. Recuperado de http://www.mintic.gov.co/portal/inicio/ Sala-de-Prensa/Noticias/14587:Las-
TIC-siguen-impactando-positivamentela-educacion-colombiana

Murphy, E., \& Sunthonkanokpong, W. (2019). Quality, equity, inclusion and lifelong learning in pre-service teacher education. Journal of Teacher Education for Sustainability, 21(2), 91104. https://doi.org/10.2478/jtes-20190019

Otero-Bahamon, S. (2019). Subnational Inequality in Latin America: Empirical and Theoretical Implications of Moving beyond Interpersonal Inequality. Studies in Comparative International Development, 54(2), 185-209. https://doi.org/10.1007/s12116-01909281-6

Roemer, J., \& Trannoy, A. (2016). Equality of opportunity: Theory and measurement. Journal of Economic Literature, 54(4), 1288-1332. https://doi.org/10.1257/jel.20151206

Ruiz, F. (2017). TIC en educación primaria: una propuesta formativa en la asignatura didáctica de la medida basada en el uso de la tecnología. Tendencias Pedagógicas, 30, 53-70.

UNESCO. (2008). Estándares de competencia en TIC para docentes. Revista Iberoamericana de Evaluación Educativa, 3(1), 202-217. 